\title{
Research on the Influence of Chinese on the Inheritance and Path of Yi Language
}

\author{
Yihong Jiejue"
}

Yi Culture Research Center of Xichang University, Sichuan, China, 615000

Abstract. There are many ethnic groups in our country, and the Yi ethnic group has formed its own language in history. Like other languages, Yi language also carries the nation's historical, cultural and intellectual achievements, and is an important object for studying the Yi people and even the Chinese nation. Modern society is developing rapidly, and in the development of the times and opening up, the use of other minority languages is gradually declining due to their limitations. At present, the huge influence of Chinese is also of great significance in the inheritance of Yi language.

Keywords: Chinese; Yi Language; Inheritance

\section{Introduction}

Language is not only the carrier of culture, but also the business card of culture. Only when it communicates and integrates with other languages can language be richer and have a long history. There are many ethnic groups in our country, and there have been many national integrations in history ${ }^{[1-3]}$. In the common life process, various languages also have mutual influence and reference. Among them, Chinese, as the most widely spoken and widely used language, has the greatest impact on the languages of other ethnic minorities and the most far-reaching. As a common language, Chinese is often used as an object of reference and introduced into the language system of ethnic minorities ${ }^{[1-6]}$. Yi language is not immune to the development of the historical situation. ${ }^{[1]} \mathrm{With}$ the extensive exchanges of social commodities and talents and the overall development of economy and transportation, the contact between Yi language and Chinese through various means has increased correspondingly, and the communication in writing is also increasing day by day, and more frequent and close. Language is an incredible cultural symbol ${ }^{[7]}$. Through the application of language in various forms, communication can be unimpeded, culture is spread, and development and prosperity are brought about. At the same time, the consistency of language also brings the same sense of cultural identity. ${ }^{[2]}$ The Yi language was first used in the scope of activities of the Yi people. With the increasing population flow, the number of people using Yi language is declining. The decline of the Yi language is gradually revealed in the historical changes, and its protection and inheritance are gradually on the agenda. It is of great significance to safeguard the development of the Yi people.

\section{Overview of the use of Yi language}

Through the investigation of society and some subject research organizations, combined with the author's research data collection in related aspects, the author has made the following understanding and generalization of the current situation of Yi language use. Figure 1 shows the current composition of the Yi language population.

Copyright () 2020 Yihong Jiejue

doi: $10.18282 /$ le.v9i7.1528

This is an open-access article distributed under the terms of the Creative Commons Attribution Non-Commercial License

(http://creativecommons.org/licenses/by-nc/4.0/), which permits unrestricted non-commercial use, distribution, and reproduction in any medium, provided the original work is properly cited. 


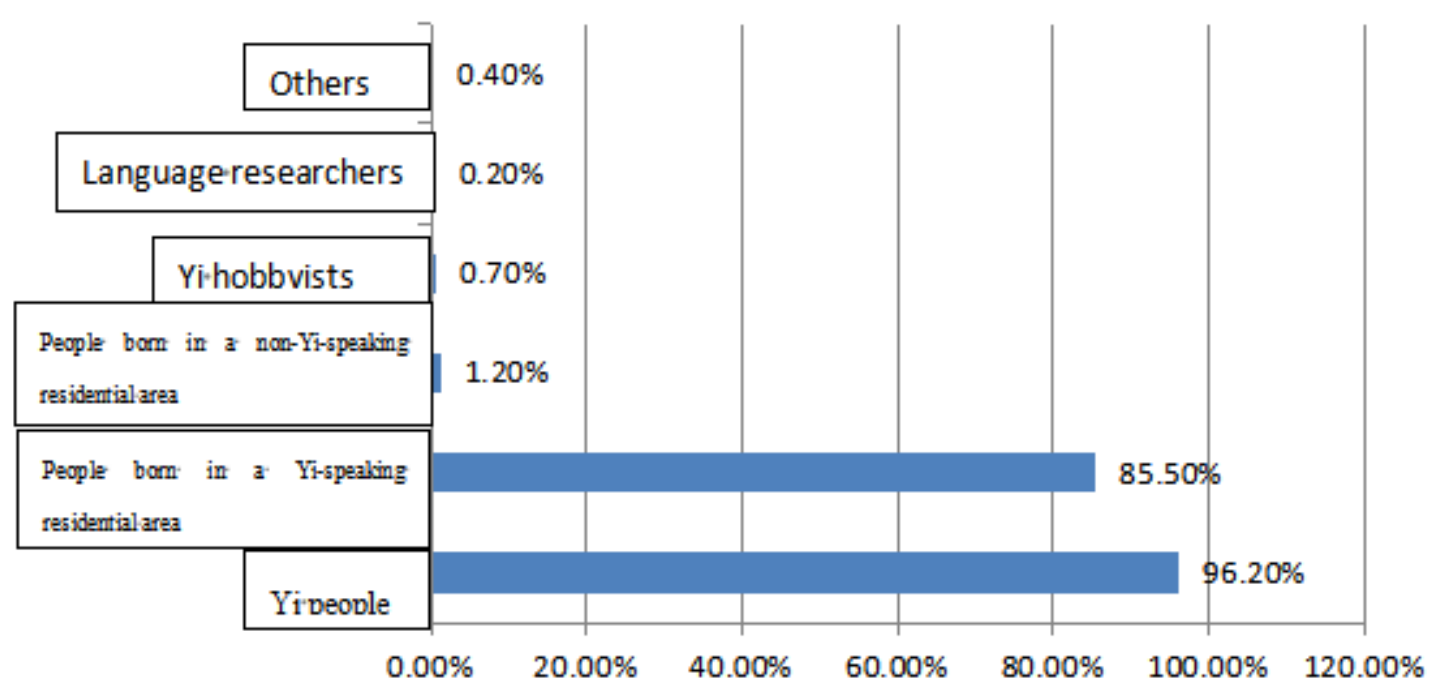

Figure 1. Proportion of people in different categories among Yi language speakers.

\subsection{First mother tongue}

The mother tongue is the easiest language for many people to master and use. It is inseparable from the timing of contact and the allocation of languages in the human brain, and is not within the scope of this article ${ }^{[3]}$ According to some conclusions from the medical and linguistic theory circles, the majority of people who are proficient in Yi language are people who have Yi language as their first mother tongue, and most of them are in areas where Yi people are relatively concentrated. Many people acquire a second mother tongue, Chinese, after entering school, and gradually enter the state of communicating with the outside world in Chinese.

\subsection{Skilled people and areas}

The data and conclusions obtained from various sources all support that the Yi people are the most proficient in Yi language, and their living area is the most widely and proficient area in Yi language ${ }^{[4]}$ Among these people and regions, Yi language plays an extremely important role in social activities. It can even be said that the Yi language is a bridge between various relationships within Yi society.

\subsection{Usage scenarios}

At present, the Yi language is in an awkward situation and is becoming a minority. Between the Yi people's distribution area and the Yi people, Yi language is still the most commonly used language in many daily scenes. Among them, the elderly and children only master the Yi language. Most Yi people speak Chinese and Yi. Bilingual users. ${ }^{[5]}$ They have the conditions to choose the language to use according to the communication situation, and there will even be scenes where Chinese and $\mathrm{Yi}$ are used interchangeably to enhance the effect of communication.

\section{Briefly describe the development of Yi language}

The users of Yi language are mainly distributed in the southwest part of our country. It has a rich linguistic level. There are also different dialects, sub-dialects, and internal dialects in different regions, which are as complicated as other languages. In modern society, human activity has increased. In addition to the daily communication of Yi people and their living areas, Yi language has almost replaced Chinese in its social functions.

\subsection{Getting started}

On the opposite side of language studies, my country is also a rising star. Previously, scholars had extremely limited special research on Yi language. It was not until foreign countries took the lead in language research to have breakthroughs in new theories and methods that led to profound changes in domestic sociolinguistic research ${ }^{[6]}$ The focus of the research was from written to spoken And the transfer of daily expression, from the general language to the minority representative language. During a long period of research, experts and scholars have gradually discovered the relationship between the Yi language and the social development of the Yi people, and discovered the social attributes of the language. 


\subsection{Development}

After the efforts and development of scholars in the previous period, our country has also accumulated rich experience in the study of minority languages, formed its own ideas, and made many new discoveries. As an intangible cultural heritage, Yi language has attracted the attention and research of many domestic scholars. The research on it includes but not limited to social function, language structure, pronunciation norms and included historical and cultural composition. ${ }^{[7]}$ The continuous publication of relevant works on Yi language research has made the Yi language-related database increasingly enriched, which is more conducive to the systematic and standardized development of Yi language research, and also provides detailed information for the influence of Chinese on Yi language and the formulation of Yi language inheritance strategies .

\section{The inevitability of Chinese influence on Yi language inheritance}

\subsection{The impact of developments and changes in the domestic and international situation}

Especially since the reform and opening up, our environmental conditions at home and abroad have undergone unexpected changes. The market economy has unprecedented requirements for language communication. In an open international environment and under the influence of domestic policies, ethnic minorities have opened up and developed on an equal footing with the Han people. Their lives have improved with each passing day, and their spiritual and cultural levels have also risen. However, in the process of increasing the degree of foreign exchanges, the Yi people's dependence on Chinese has gradually increased, and the Yi language has suffered from social neglect due to its social function being restricted by itself.

\subsection{Requirements for national development and national unity development}

Chinese is the common language of our country. For the convenience of communication and thorough familiarity, learning Chinese is something that all regions and all ethnic groups should pay attention to. Only by proficiently mastering the usage of Chinese and being able to understand the meaning of Chinese words and sentences well can the management of the country operate efficiently, and the various ethnic groups can understand each other, tolerate each other, and help each other, forming a united and upward overall force.

\subsection{The result of the weak awareness of the Yi people's mother tongue}

my country's history is ancient, but its development has been very slow for thousands of years. The family units mostly exist in a self-sufficient way for men farming and women weaving, or a combination of planting, hunting, and gathering has long existed in some more backward minority areas. Similar to the living conditions of primitive society, people's material living standards have been low for a long time and suffer from hunger and cold. Therefore, after they have benefited from social and economic development, they will concentrate on Chinese, which has a more powerful social function, thereby ignoring the importance of national language in national development and embarking on the path of "partial science". As this cycle continues, the native languages of ethnic minorities, including the Yi language, have encountered the biggest inheritance dilemma in history.

\section{The influence of Chinese in the inheritance of Yi language}

\subsection{Provide words that are not in the Yi language}

Some linguists once said that language is not self-sufficient for any nation, and it often needs to learn other languages to enrich itself. Especially in modern times, many new things emerge in an endless stream, and the development of language must adapt to it. While Chinese is advancing with the times and enriching its own language library, it also provides some languages that Yi language lacks in the process of communicating with Yi language, so that the connection between the two is more precise and the communication is more smooth.

\subsection{Synonymous reference increases the richness of Yi language}

Because Chinese has stronger versatility, better social functions, and more detailed language usage scenarios and division of labor, the Yi language will not only borrow Chinese vocabulary to fill in the gaps, but also learn from 
Chinese words with the same meaning to expand its language. It also makes it more comprehensible with Chinese.

\subsection{Increase the basic vocabulary of Yi language}

With the deepening of the influence of Chinese, many Chinese vocabularies have been used by the Yi language for a long time, and they have been integrated into the Yi language to carry out new transformations or give innovative meanings, thus becoming the basic vocabulary of the Yi language.

\section{Difficulties facing Yi language in inheritance}

\subsection{The impact of social and economic prosperity}

In the past few decades, we have worked hard on the correct line, social and economic development, and spiritual life have been greatly enriched. A big problem that has been brought about is the phenomenon of "ideological decline" among many people, that is, the supremacy of individualism. The concept of money first is very popular. The relationship between language work and the market economy is not very close. It requires researchers to resist the temptation of external materials and calm down for a long time to study hard. Many people give up halfway because there is no "money" or dare not get started. This is a set of contradictions that are not conducive to the inheritance of Yi language due to the unbalanced development between Yi language development and economy.

\subsection{The danger of "fracture" in Yi language inheritance}

my country has been open to the outside world for more than 40 years. Not only is our own language and culture going out step by step to show the real China to the world, at the same time, foreign languages and cultures are also pouring into our country continuously. Most ethnic minority areas have low economic openness and even lower cultural openness. Because of the conservativeness in history, the Yi language pales in comparison with Chinese in dealing with foreign language and cultural reactions. The Chinese language can easily deal with foreign languages, assimilate, or follow the good, while the Yi language is caught in a dilemma. Due to the extremely limited scope of application, the absorption of foreign languages by Yi language is likely to cause a break in its own language inheritance, and rejection of foreign languages is to isolate itself. Over time, it still cannot escape the fate of inheritance break.

\section{Consideration of the inheritance path of Yi language}

\subsection{Start with the study of Yi language}

The Yi language has been passed down among Yi people for thousands of years as a social communication tool, but the modern research on the Yi language has only happened in recent decades. Nowadays, the Yi language is gradually fading out of the stage of communication. If it is allowed to fend for itself, it will not only be the loss of the Yi people and Yi culture, but also the loss of Chinese and even the world culture. As a special research object, there are two clear benefits. One is to collect and protect more Yi language information materials through research; the other is that research helps to explore and clarify the true social value of Yi language, thereby triggering a wide range of people. attention.

\subsection{Establishment of Yi language education courses}

To protect and inherit the Yi language, first set up special Yi language courses in all levels of education in areas where Yi language is routinely used (such as Yi people's residential areas and areas where Yi language is the mother tongue), forming a special specialty, and focusing on Yi language teachers The cultivation of Yi language also focuses on the cultivation of students majoring in Yi language. With the maturity of the courses, the Yi people will not only become familiar with the Yi language, but also attract some Yi language lovers to come for special learning. By setting up the Yi language major, the goal of inheriting both self-inheritance and external inheritance can be achieved.

\section{Conclusion}

As the official lingua franca, Chinese has a rich language database, complete social functions, and a wide range of uses. It has a profound impact on minority languages including Yi language. Although the social function of Yi 
language is gradually weakening, its historical and cultural value is endless. This article explores the method of economic development and protection and inheritance of Yi language, which is an urgent task to solve the difficulty of Yi language inheritance.

\section{References}

1. Luo Niuniu. Thoughts on the inheritance and protection of Yi Language[J]. Guizhou Ethnic Studies, 2015(3):235238.

2. Yang Lan. Investigation on the language life of the Yi people in the process of urbanization [D]. 2019.

3. Zhang Lu. On the Influence of Chinese on the Yi People's language inheritance and research on inheritance paths[J]. Guizhou Ethnic Studies, 2015(2): 202-205.

4. Ma Xinguo. Introduction to Yi language and culture [M]. Yunnan Nationalities Publishing House, 2012.

5. Ma Junjie. Research on the teaching value and inheritance path of Yi nationality dance[J]. Artwork Jian, 2018, 000(021): P.297-298.

6. Zhu Yuanfu. Research on the endangerment trends and countermeasures of the Yi People's language and characters in wuding county [C]//Proceedings of the Second Yi Studies Symposium in the four provinces (districts) of Yunnan, Sichuan, Guizhou and Guizhou. 2011.

7. Hu Hongbo. Research on the problems and countermeasures of chinese language learning in primary schools in $\mathrm{Yi}$ Nationality Regions [J]. Speed Reading (1st 10th), 2016(7). 